QUANTUM ANOMALOUS HALL EFFECT Honeycomb recipe

npj Comput. Mater. 3, 39 (2017)

When a magnetic field is applied perpendicular to the current flow in a conductor, a transverse voltage appears due to the well-known Hall effect. If the conductor has ferromagnetic ordering, then this voltage can emerge without the need for an external magnetic field. The quantum version of this anomalous Hall effect is topological in nature; unlike its ordinary counterpart whose origins date back to the nineteenth century, the quantum anomalous Hall effect is extremely difficult to realize experimentally. Christian Crisostomo and colleagues have now predicted that chemical functionalization could provide new platforms for developing robust quantum anomalous Hall phases.

By magnetically doping topological insulator thin films, quantum anomalous Hall states have already been realized, but only at very low temperatures. Using first-principles calculations, Crisostomo et al. showed that chemical functionalizing with hydrogen or nitrogen atoms could enable certain bismuthbased materials with a honeycomb lattice to host quantum anomalous Hall phases with bandgaps that are much larger than current realizations, making them more stable. As such phases support chiral edge states that are fully spin-polarized, this could provide new opportunities for spintronics and low-power electronic devices.

\section{MANY-BODY LOCALIZATION}

\section{Going long}

Phys. Rev. X (in the press); preprint at

http://arxiv.org/abs/1705.06290

Sometimes, even physicists succumb to folklore. This typically arises when plausible, sometimes even rigorous arguments are widely understood to mean a particular phenomenon cannot arise under certain conditions. The 'impossibility' of isolating two-dimensional sheets of carbon is a prime example from the pre-graphene days.

A similar folk theorem seems to have taken hold in the study of the nonequilibrium quantum dynamics of manybody systems, the phenomenon known as many-body localization (MBL): based on perturbative arguments, it is generally thought that MBL is only possible in systems with short-range interactions.

Now, Rahul Nandkishore and Shivaji Sondhi have put forward a set of nonperturbative arguments to show that MBL can also arise with long-range interactions. The key idea is that they can drive the system into a non-trivial correlated phase, which is naturally described in terms of emergent degrees of freedom with only short-range interactions. The arguments are most rigorous in one dimension, but can be extended to two and three dimensions as well. MBL may therefore be more widespread than previously thought.

\section{PROTON PUZZLE}

\section{Agreement in disagreement Science 358, 79-85 (2017)}

Seven years ago, measurements of muonic hydrogen - an exotic atom with a muon instead of an electron - yielded a radius of the proton significantly smaller than expected. The result was very puzzling, but follow-up measurements only confirmed it, deepening the mystery. Now Axel Beyer and colleagues have revisited the measurements of the proton radius using the $2 s-4 p$ transition frequency in regular hydrogen. The new result is in line with the values from muonic hydrogen
GAS DETECTION

\section{Sensor extraordinaire}

A widespread approach to gas sensing uses materials whose electrical resistivity changes on exposure to a target analyte. Such conductometric solid-state sensors are versatile and relatively inexpensive, but they typically lack chemical selectivity. This could be changed with sensor materials that show not only an electric but also a strong magnetic response to components of a gas, as Alexander Gerber and colleagues have now demonstrated.

The idea of using changes in magnetic properties for gas detection is not new, but Gerber et al. provide a practical route to exploiting such a concept. Their sensor is based on cobalt-palladium thin films. The material displays a strong extraordinary (or anomalous) Hall effect, brought about by the broken time-reversal symmetry due to spin-orbit coupling. And the alloy dissolves hydrogen. Combine these qualities and the additional magnetic response makes the sensor two orders of magnitude more sensitive for hydrogen than when only changes in electrical conductance are considered, as Gerber and his co-workers showed in a series of experiments, highlighting the promise of complementing conductometric sensing with this spintronic effect.

spectroscopy and hence finds itself at odds with the accepted value of the proton radius.

Beyer et al. used a cryogenic beam of hydrogen atoms in one of the hyperfine ground states, which they excited to the $4 p$ state. They measured the transition frequency from which they derived the proton radius and the value of the Rydberg constant, which also disagrees with the figures in the literature - by more than three standard deviations. Despite the excellent accuracy, the new values do not offer a solution for the proton puzzle, but rather call for more precision measurements.

\section{BIOMECHANICS}

\section{Split sites}

Proc. Natl Acad. Sci. USA 114, 10888-10893 (2017)

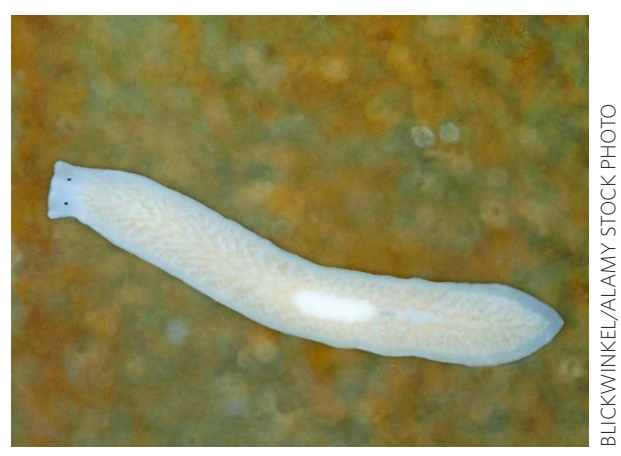

Planarians (pictured) are intriguing creatures: these little freshwater worms reproduce by ripping themselves in two - literally separating head from tail — and then regenerating the bit that each half lost. But they're also rather shy, and seldom actually commit to the task of reproducing. These quirks have frustrated planarian research for centuries, and the seemingly random location of division along the body axis has come to be thought of as entirely unpredictable. But now, Paul Malinowski and colleagues revealed that pinpointing the fission site is simply a matter of mechanics.

Decapitated planarians are known to divide more frequently, so Malinowski and co-workers exploited this tendency to ramp up their data collection. A mechanical model comprising a thin elastic shell in place of the planarian's head succeeded in reproducing the body dynamics preceding fission. And a statistical analysis revealed that the time between fission events was correlated with the location of division relative to the pharynx, which the worms use to ingest food. Variable stays between divisions translated into variable fission sites, due to the sheer mechanics of rupturing around a large muscle.

Written by Luke Fleet, Iulia Georgescu, Abigail Klopper, Andrea Taroni and Andreas H. Trabesinger. 